\title{
Diverse Evolutionary Trajectories for Small RNA Biogenesis Genes in the Oomycete Genus Phytophthora
}

\author{
Stephanie R. Bollmann ${ }^{1+}$, Yufeng Fang ${ }^{2,3}$, Caroline M. Press ${ }^{1}$, Brett M. Tyler ${ }^{2}$ and \\ Niklaus J. Grünwald ${ }^{1,2 *}$ \\ ${ }^{1}$ Horticultural Crop Research Unit, USDA-Agricultural Research Service, Corvallis, OR, USA, ${ }^{2}$ Department of Botany and \\ Plant Pathology and Center for Genome Biology and Biocomputing, Oregon State University, Corvallis, OR, USA, \\ ${ }^{3}$ Interdisciplinary Ph.D. Program in Genetics, Bioinformatics and Computational Biology, Virginia Tech, Blacksburg, VA, USA
}

OPEN ACCESS

Edited by:

Hua Lu,

University of Maryland Baltimore

County, USA

Reviewed by:

Mark Gijzen,

Agriculture and Agri-Food Canada,

Canada

Ramesh Raju Vetukuri,

SLU, Sweden

*Correspondence:

Niklaus J. Grünwald

nik.grunwald@ars.usda.gov

${ }^{\dagger}$ Present Address:

Stephanie R. Bollmann,

Department of Integrative Biology, Oregon State University, Corvallis, OR,

Specialty section:

This article was submitted to Plant Biotic Interactions,

a section of the journal

Frontiers in Plant Science

Received: 21 January 2016 Accepted: 22 February 2016 Published: 15 March 2016

Citation:

Bollmann SR, Fang Y, Press CM, Tyler BM and Grünwald NJ (2016) Diverse Evolutionary Trajectories for Small RNA Biogenesis Genes in the

Oomycete Genus Phytophthora.

Front. Plant Sci. 7:284.

doi: 10.3389/fp/s.2016.00284
Gene regulation by small RNA pathways is ubiquitous among eukaryotes, but little is known about small RNA pathways in the Stramenopile kingdom. Phytophthora, a genus of filamentous oomycetes, contains many devastating plant pathogens, causing multibillion-dollar damage to crops, ornamental plants, and natural environments. The genomes of several oomycetes including Phytophthora species such as the soybean pathogen $P$. sojae, have been sequenced, allowing evolutionary analysis of small RNA-processing enzymes. This study examined the evolutionary origins of the oomycete small RNA-related genes Dicer-like (DCL), and RNA-dependent RNA polymerase (RDR) through broad phylogenetic analyses of the key domains. Two Dicer gene homologs, $D C L 1$ and DCL2, and one RDR homolog were cloned and analyzed from P. sojae. Gene expression analysis revealed only minor changes in transcript levels among different life stages. Oomycete DCL1 homologs clustered with animal and plant Dicer homologs in evolutionary trees, whereas oomycete DCL2 homologs clustered basally to the tree along with Drosha homologs. Phylogenetic analysis of the RDR homologs confirmed a previous study that suggested the last common eukaryote ancestor possessed three RDR homologs, which were selectively retained or lost in later lineages. Our analysis clarifies the position of some Unikont and Chromalveolate RDR lineages within the tree, including oomycete homologs. Finally, we analyzed alterations in the domain structure of oomycete Dicer and RDR homologs, specifically focusing on the proposed domain transfer of the DEAD-box helicase domain from Dicer to RDR. Implications of the oomycete domain structure are discussed, and possible roles of the two oomycete Dicer homologs are proposed.

Keywords: dicer, RDR, evolution, Phytophthora, small RNA, stramenopile

\section{INTRODUCTION}

Gene regulation through the action of small RNAs is ubiquitous among eukaryotes and is well characterized in plants, animals, and fungi but not in other eukaryotic lineages (Ghildiyal and Zamore, 2009). The three most prevalent types of small RNAs are short interfering RNAs (siRNA), microRNAs (miRNA), and PIWI-interacting RNAs (piRNA), with the siRNA pathway being common to all investigated eukaryotic kingdoms. SiRNAs act in silencing of repetitive sequences 
such as transposons and in antiviral defense (Hamilton and Baulcombe, 1999). miRNAs regulate many biological processes, with defects in miRNAs causing severe phenotypes such as autoimmune disease and cancer (Alemdehy et al., 2015; Mehta et al., 2015). miRNA expression is often specific to a tissue or developmental stage (Lee et al., 1993; Wightman et al., 1993). PiRNAs, which are specific to metazoans, are required for germline stem cell maintenance and promotion of cell division (Aravin et al., 2001; Vagin et al., 2006).

To date, small RNAs have been described for plants, animals, fungi, and some protists, yet little is known about small RNA biology in the Stramenopile kingdom, which includes golden-brown algae, diatoms, brown algae, and oomycetes. Many oomycetes, including those in the genus Phytophthora, are devastating plant pathogens, affecting diverse hosts and causing multibillion dollar damage to crops, ornamental plants, and natural environments. A number of oomycetes are also pathogens of aquatic hosts, such as the fish pathogen Saprolegnia parasitica. Notable Phytophthora species include Phytophthora infestans that infects potatoes and tomatoes and caused the Irish potato famine, $P$. sojae that infect soybeans, and $P$. ramorum that causes sudden oak death (Tyler, 2007; Fry, 2008; Grünwald et al., 2008). The genomes of these three Phytophthora species have been sequenced (Tyler et al., 2006; Haas et al., 2009), as well as the more distantly related oomycetes Hyaloperonospora arabidopsidis (Baxter et al., 2010), Pythium ultimum (Lévesque et al., 2010), Albugo candida (Links et al., 2011), and S. parasitica (Jiang et al., 2013), opening the door to investigation of oomycete small RNA pathways through comparative genomic approaches.

Small RNA biogenesis requires processing by two key enzymes: Dicer [or dicer-like (DCL) in non-metazoans] and RNA-dependent RNA polymerase. Briefly, siRNAs originate from an inverted repeat transcript, sense/anti-sense transcription of the same template, or the action of an RNA-dependent RNA polymerase (RDR) on an ssRNA template (Makeyev and Bamford, 2002; Ghildiyal and Zamore, 2009). All siRNAs are processed by Dicer (DCR), an RNase III endonuclease, with the assistance of a dsRNA-binding protein (Bernstein et al., 2001). DCR cleavage yields an RNA duplex with $5^{\prime}$ phosphates and a two nucleotide overhang on the $3^{\prime}$ end. Guide and passenger strands are determined by the relative thermodynamic stabilities of the $5^{\prime}$ ends, and the guide strand is loaded into the RNAinduced silencing complex (RISC) with an Argonaute (AGO) protein and in some cases auxiliary proteins (Tabara et al., 1999; Hammond et al., 2000; Zamore et al., 2000). RISC complexes containing siRNAs repress their target mRNAs through cleavage by AGO, which leads to the production of secondary siRNAs, a process known as RNA interference (RNAi). Unlike siRNAs, miRNAs do not require an RDR for biogenesis. miRNAs originate from a primary miRNA transcript (pri-miRNA), which forms a secondary stem and loop structure. The mature miRNA is formed through two processing steps by an RNase III endonuclease and dsRNA-binding domain partner protein (Denli and Hannon, 2003). In animals, the pri-miRNA is processed in the nucleus by Drosha, producing a pre-miRNA with a $3^{\prime}$ overhang (Lee et al., 2003). The pre-miRNA is then exported to the cytoplasm, where it undergoes a second processing step by Dicer to form the mature miRNA/miRNA* (e.g., guide/passenger) duplex. The miRNA guide strand, similar to siRNAs, is chosen through thermodynamic selection and loaded into a RISC complex. In plants, DCL1 performs both processing steps of the pri-miRNA in the nucleus (Park et al., 2002; Reinhart et al., 2002; Schauer et al., 2002). The mechanism of miRNA target regulation, by either cleavage or translational repression, is determined by which AGO binds the miRNA and the degree of complementarity to the target mRNA. Although miRNAs have been observed in plants and animals, no conservation of the miRNA genes has been found across kingdoms. Canonical miRNAs have not been isolated from fungi, but a recent study by Braun et al. (2010) described putative miRNAs in Toxoplasma gondii, an apicomplexan in the Chromalveolate supergroup. One miRNA family was found to be conserved in all published Phytophthora genomes, with evidence for canonical target mRNA cleavage for two predicted targets of the miRNA in P. sojae (Fahlgren et al., 2013).

Dicer (DCR) homologs, which include Dicer-like (DCL) proteins, are well conserved across many eukaryotic kingdoms, yet the number of homologs within a taxonomic group is quite variable. Dicers have been studied most extensively in animals, plants, and fungi, and limited numbers of Dicer homologs have been identified bioinformatically in other eukaryote lineages. Trypanosoma brucei, from the Excavata supergroup, contains two Dicer-like proteins, although the structure is unusual (Shi et al., 2006). From the Chromalveolate supergroup, diverse numbers of small RNA-related genes have been identified from a limited number of organisms. The apicomplexan T. gondii has a complex small RNA landscape, yet contains one homolog each of DCR, RDR, and AGO (Braun et al., 2010). The ciliate Tetrahymena thermophila harbors three DCR homologs (Lee and Collins, 2007), while Paramecium tetraurelia has seven annotated DCR/DCL homologs, with a proportion of each potentially being nonfunctional (Lepère et al., 2009). Higher metazoans have one Dicer homolog, insects and Cnidarians have two Dicers, and basal animals (Placozoa and Porifera) have up to five Dicers. Phylogenetic analysis of this larger set of metazoans by de Jong et al. (2009) indicates an ancient duplication event of a "Proto-Dicer" gene, with only Placozoans retaining both versions. The multiple Dicers in the basal metazoans are thought to be the result of lineage-specific duplications. Plants have also gained multiple DCL homologs through duplication, and studies of these paralogs reveal differentiation of function, including miRNA and siRNA specificity and some with roles in viral defense (Park et al., 2002; Xie et al., 2004, 2005; Dunoyer et al., 2005; Gasciolli et al., 2005; Kasschau et al., 2007). This suggests that plants use their specialized RNA machinery for immune defense, and may also explain the higher number of Dicer homologs in basal metazoans (de Jong et al., 2009). All Dicers have two RNase III domains ( $a$ and $b$ ) in the Cterminus. Other domains commonly found include the DEADbox helicase, helicase C, dsRBD, PAZ, and dsrm. The PAZ, RNase III, and dsrm domains are responsible for dsRNA-binding and cleavage (Liu et al., 2009). PAZ binds the $3^{\prime}$ two-base overhang of dsRNA, and is connected to RNase IIIa by a long $\alpha$-helix whose distance functions as a molecular ruler to determine the length 
of the cleaved RNA (MacRae et al., 2006). RNase III domains can also directly bind the PIWI domain of AGO (Tahbaz et al., 2004). The dsRBD is involved in siRNA/miRNA strand selection (Dlakić, 2006).

RDR has also been conserved across eukaryotic kingdoms, although very few animal RDR homologs have been identified, and variation in the number of homologs among different groups is also observed. A study by Cerutti and Casas-Mollano (2006) reconstructed the putative small RNA gene complex of the last common eukaryotic ancestor. Their phylogenetic analyses across five of the eukaryotic supergroups suggested a monophyletic origin for DCR and RDR, while more-specific RNAi-mediated pathways were independently acquired in some lineages and some RNAi pathways or factors were lost in other lineages. For example, an RDR homolog is generally not present in species that can produce dsRNA by other methods. The evolutionary relationships of the RDR tree were not clear, however, potentially due to the use of neighbor-joining trees for analysis (Cerutti and Casas-Mollano, 2006). A more comprehensive analysis by Zong et al. (2009), including plants, fungi, invertebrate animals, and other eukaryotes proposed an alternate model where the eukaryotic ancestor had three copies of RDR: $\alpha$, $\beta$, and $\gamma$. Phylogenetic analysis and patterns of conserved protein sequence motifs provided evidence for the three ancient homologs, although the only defined domain common to all RDR homologs was the RNA-dependent RNA polymerase (RDRP) domain. The variability in homolog numbers among different organisms was suggested to result from further duplication events after divergence of the major groups, with the $\alpha$ - and $\gamma$ type RDR homologs showing greater expansion. The duplication of RDR homologs, similar to Dicer, has allowed differentiation of function, as has been demonstrated in plants (Dalmay et al., 2000; Yu et al., 2003; Xie et al., 2004; Herr et al., 2005; Kasschau et al., 2007). Although the analysis by Zong et al. (2009) did not result in strong support values for the relationships of other eukaryotic clades to plant, animal or fungal clades, all of the homologs from other eukaryotic groups were clustered within each of the three putative ancient clades.

Previous Phytophthora studies have provided evidence for functional RNA interference pathways, both post-transcriptional gene silencing and transcriptional gene silencing. Initial reports of transgene inactivation (Judelson and Whittaker, 1995) were followed by directed transgene and endogenous gene silencing by sense, anti-sense, and promoter-less constructs (van West et al., 1999; Gaulin et al., 2002; Latijnhouwers and Govers, 2003; Blanco and Judelson, 2005), or alternately a hairpin construct to induce silencing of an endogenous gene (Judelson and Tani, 2007). The presence of 21-nucleotide RNAs from both the sense and antisense strands of the transgene were observed in partially silenced lines (Ah-Fong et al., 2008). The method of transgene silencing was proposed to initially be through post-transcriptional gene silencing using small RNA, followed by transcriptional gene silencing through chromatin modification (Ah-Fong et al., 2008). The study also bioinformatically identified small RNA-processing enzymes from P. infestans, including one Dicer-like (DCL1), one RDR, and five AGO homologs. A study of the expression of these and other possible small RNA-related proteins provided evidence for DCL1 and AGO1,2 playing a role in transgene silencing (Vetukuri et al., 2011). An alternate domain structure of $P$. infestans RDR containing a DCR-like DEAD-box helicase was observed, similar to the structure seen in Dictyostelium (Vetukuri et al., 2011). High-throughput sequencing of P. infestans small RNAs revealed peaks at sizes of 21- and 25/26-nt (Vetukuri et al., 2012). Additionally, there was evidence for DCL1 having a role in production of 21-nt size small RNAs. This differs from the diatoms Phaeodactylum tricornutum and Thalassiosira pseudonana, which also have one DCR-like protein, but small RNA sequencing data revealed only one peak of small RNAs at 22-nt (Huang et al., 2011; Norden-Krichmar et al., 2011). In $P$. infestans, similar to $P$. sojae and $P$. ramorum, there are in fact two conserved DCL genes, one RDR, and five AGO genes, with $P$. sojae and $P$. ramorum having additional AGO genes and pseudogenes (Fahlgren et al., 2013). The small RNA peaks at 21- and 25/26-nt were also confirmed for those three species (Fahlgren et al., 2013). One conserved miRNA family was discovered in the three species, and modified $5^{\prime}$ RACE confirmed canonical cleavage of two predicted targets (Fahlgren et al., 2013).

The goal this study was to conduct a comprehensive analysis of the evolution of the DCR and RDR genes in oomycetes. We cloned and annotated the small RNA biogenesis genes DCL1, $D C L 2$, and $R D R$ in $P$. sojae and analyzed their expression in various life stages. We experimentally validated nuclear targeting of DCL1 and DCL2. We performed phylogenetic analyses of oomycete and other Stramenopile DCLs and RDRs compared to those from a broad range of eukaryotic kingdoms using the key conserved domains of each protein. Domain transfer of the DEAD-box helicase domain from DCR to RDR in oomycetes is also discussed, as well as a proposed function for the two DCR homologs.

\section{MATERIALS AND METHODS}

\section{Selection of Eukaryotic Genes for Phylogenetic Analyses}

Dicer and RDR homolog sequences were initially identified in the NCBI database (www.ncbi.nlm.nih.gov) using TBLASTN searches. Various queries were used, including full-length sequences or RNase III/RDRP domain sequences from species such as Homo sapiens, Trichoplax adhaerens, Arabidopsis thaliana, and Phytophthora sojae. Species with partial or fully-sequenced genomes were selected from all eukaryotic supergroups with the exception of Rhizaria. DCR and RDR homologs identified in previous phylogenetic analyses (de Jong et al., 2009; Zong et al., 2009) were included along with homologs from kingdoms that were less represented previously. Additional sequences were identified through TBLASTN searches of species-specific genome databases, and the DOE JGI database (genome.jgi-psf.org) was searched by querying specific organisms for the presence of annotated RNase III, DEAD-box helicase, or RDRP domains. Research in different organisms resulted in different naming conventions (DCR and DCL); when already annotated we retained the provided name, otherwise we used the DCR designation. 
Oomycete homolog sequences were identified at the VBI Microbial Database (currently housed at: eumicrobedb.org) and the $P$. infestans database at the Broad Institute (www.broadinstitute.org/annotation/genome/phytophthora_ infestans/MultiHome.html). Peronospora tabacina homolog sequences were originally provided by David Zaitlin, and the genome has recently been published (Derevnina et al., 2015). Genomic sequences encompassing the identified homologs were isolated and coding sequences were manually identified or confirmed using Genscan (Burge and Karlin, 1997) or FGENESH (linux1.softberry.com/berry.phtml). Protein domains were identified using a search of the Pfam database (URL; Finn et al., 2010). To be retained for subsequent analyses, a Dicer homolog minimally had to contain two RNase III domains, and an RDR homolog minimally had to contain an RDRP domain. Drosha homologs and RNase III domain-containing genes from bacteria and yeast were also identified to provide outgroups. $P$. sojae sequences have been deposited at GenBank under the following accession numbers: DCL1 (KT387801, KT387802), DCL2 (KT387803), and RDR (KT387804).

\section{Cloning and Sequencing of $P$. sojae DCL and RDR Homologs}

Total RNA was isolated from mycelium of $P$. sojae strain P6497 using TRIzol (Invitrogen, Carlsbad, CA), and mRNA was isolated from $250 \mu \mathrm{g}$ total RNA with an Oligotex column (Qiagen, Germantown, MD) cDNA was synthesized with the GeneRacer RACE Ready kit (Invitrogen) following the manufacturer's guidelines. Genscan-predicted coding sequences from genome database queries were analyzed with the Primer3 (Rozen and Skaletsky, 2000) plugin in Geneious version 8.0.2 (Kearse et al., 2012) to design primers. Primers were initially tested on genomic DNA isolated from mycelium with a FastPrep kit (MP Biomedicals, Solon, $\mathrm{OH}$ ) to confirm the predicted genome sequence and test the efficacy of the primers. Primer sequences are listed in Supplementary Table 1. All PCR products were amplified with GenScript Taq polymerase following manufacturer's guidelines: all primers were designed to use annealing temperatures between 56 and $60^{\circ} \mathrm{C}$, with an extension time of $1 \mathrm{~min}$ per $1 \mathrm{~kb}$. Amplification of the $5^{\prime}$ and $3^{\prime}$ UTRs utilized touchdown PCR, as recommended by GeneRacer guidelines, typically with 1-min extension. Internal sequence amplification typically involved $60^{\circ} \mathrm{C}$ annealing temperature and 3-min extensions. All PCR products were cloned with the TOPO-TA cloning kit (Invitrogen). A minimum of four clones were sequenced for each product with BigDye Terminator v. 3.1 Cycle Sequencing Kit on an ABI Prism 3730 Genetic Analyzer (Applied Biosystems, Carlsbad, CA) at the Oregon State University Center for Genome Research and Biocomputing. The sequences were assembled and analyzed manually using Geneious software. The full-length DCL1 and DCL2 sequences were amplified from $P$. sojae cDNA using primers DCL1F/DCL1-R (GC buffer and touchdown program from 68 to $58^{\circ} \mathrm{C}$ ) and DCL2-F/DCL2-R (standard manufacturer protocol), and Phusion ${ }^{\circledR}$ High-Fidelity DNA Polymerase (NEB) respectively. The PCR amplicons were in-frame fused with GFP by inserting into the blunt site Stu I in the plasmid pYF2-GFP (Fang and Tyler, 2016).

\section{qRT-PCR Analysis of Gene Expression}

Total RNA was isolated using TRIzol from $P$. sojae strain P6497 mycelium, zoospores, and germinated cysts prepared in six independent biological replicates. Zoospores were produced by repeated washing of 11 day-old V8-200 plates of mycelium followed by incubation overnight at $14^{\circ} \mathrm{C}$ (Tyler et al., 1996). Germinated cysts were produced from zoospores by exposure to cleared V8 medium for $1 \mathrm{~h}$. Soybean infection experiments followed the protocol of Qutob et al. (2000) in which myceliuminoculated hypocotyls were incubated at $28^{\circ} \mathrm{C}$ for $14 \mathrm{~h}$ in the light and $25^{\circ} \mathrm{C}$ for $10 \mathrm{~h}$ in the dark. $50 \mu \mathrm{g}$ of TRIzolisolated RNA from each replication were purified from DNA using the AllPrep DNA/RNA Mini kit (Qiagen) following the manufacturer's guidelines, with the addition of one DNase I treatment. cDNA was produced using the Superscript III FirstStrand Synthesis System for RT-PCR kit (Invitrogen) and purified by phenol:chloroform extraction. cDNA was quantified with a Nanodrop ND-1000 (Thermo Scientific) and equal quantities of cDNA template were added for each reaction. Primers were designed as described above. Standard quantitative real-time PCR (qRT-PCR) reactions were performed with Fast SYBR Green Master Mix (Applied Biosystems) on an ABI StepOnePlus RealTime PCR System (Applied Biosystems). Data were normalized to reference genes $\beta$-Tubulin (Ps109498) and WS41 (Ps137777; protein of the BAR-domain family) and analyzed with Bestkeeper (Pfaffl et al., 2004), REST (Pfaffl et al., 2002), and SAS software (Cary, NC).

\section{Subcellular Localization of $P$. sojae DCL Homologs}

Laser scanning confocal microscopy (Zeiss LSM 780) was used to examine the expression and subcellular localization of DCL1GFP and DCL2-GFP fusions. Living hyphae were picked from liquid V8 cultures after 2-3 days growth. Samples were stained with DAPI $\left(4^{\prime}, 6\right.$-diamidino-2-phenylindole) for $20 \mathrm{~min}$ in the dark before microscopic examination (Hardham, 2001). Images were captured using a $63 \mathrm{X}$ oil objective with excitation/emission settings (in nm) 405/410-490 for DAPI, and 488/510-535 for GFP. Images were edited by Adobe Photoshop CS4. The tonal range was increased by adjusting highlights and shadows without altering the color balance; all images were adjusted identically.

\section{Phylogenetic Analyses}

All protein sequences used are provided in Supplementary Data Sheet 7. Alignments of protein sequences were created with MAFFT (mafft.cbrc.jp/alignment/server). For broad species analyses, protein sequences of specific conserved domains were aligned as follows: individual RNase IIIa and RNase IIIb domains, RNase IIIa and RNase IIIb domains concatenated with linking sequences deleted, DEAD-box helicase domains, and RDRP domains. To reduce errors introduced by the presence of partial sequences and to moderate the computational load, amino acid positions which were absent in a majority of the analyzed species were removed from the alignment (Baurain et al., 2010). Drosha 
homologs were included in both RNase III analyses, as they share the same double domain structure as Dicer homologs. Bacterial ribonuclease (Rnc), Saccharomyces castellii DCR1 (Drinnenberg et al., 2009), and Neurospora crassa MRPL3 (Lee et al., 2010) each contain only one RNase III domain, and so were limited to the single-domain comparison analysis. Nomenclature of the identified genes followed Zong et al. (2009). Species are identified in the phylogenetic trees with an abbreviated name in the format "Gens" (the first three letters of the genus name followed by the first letter of the species name). Gene number designations do not necessarily follow orthology. Previous designations, either from the genome database or a previous publication (de Jong et al., 2009; Zong et al., 2009), were maintained; otherwise the number assignment was arbitrary when there was no previous reference.

Condensed domain alignments were used for Bayesian phylogenetic analyses using MrBayes-3.1.2 (Huelsenbeck and Ronquist, 2001; Ronquist and Huelsenbeck, 2003). All analyses shared common settings: mixed amino acid model of evolution with invariant gamma rates, unconstrained branch lengths, sample frequency of 50, 2 runs with 4 chains each, temperature of 0.2 , and diagnostic frequency of 1000 . The concatenated $\mathrm{RNase} \mathrm{III} / \mathrm{b}$ analysis ran for 32 million generations with a final burn-in of $25 \%$, the RNase III individual domain analysis ran for 32 million generations with a final burn-in of $75 \%$, the DEAD-box helicase analysis ran for 32 million generations with a final burn-in of 75\%, and the RDRP analysis ran for 32 million generations with a final burn-in of $75 \%$. Conservation plots were produced from the condensed DEAD-box helicase domain MAFFT alignment using Geneious software (Biomatters; www.geneious.com).

\section{RESULTS}

\section{Cloning and Annotation of $P$. sojae DCR and RDR Homologs}

P. sojae homologs of DCR and RDR were initially identified bioinformatically from the VBI Microbial Database (currently housed at: eumicrobedb.org). We isolated cDNA from P. sojae mycelium and amplified overlapping segments across the length of each gene, including the use of $5^{\prime}$ RACE and $3^{\prime}$ RACE, to confirm the predicted gene structures and annotate the $5^{\prime}$ and $3^{\prime}$ UTR regions (see Supplementary Figure 1 for primer map and Supplementary Table 1 for primer information). Figure 1 shows the annotated gene structures along with conserved protein domains as predicted by Pfam. All three genes produce short UTRs: the $5^{\prime}$ UTRs ranged from 32 to 52 bases in length, and the $3^{\prime}$ UTRs ranged from 35 to $140 \mathrm{bp}$ in length. Of the three annotated genes, DCL2 contains two introns that are 71 and 83 bases in length. The GC content of the transcribed sequences is $55.2 \%$ (DCL1), 55.6\% (DCL2), and 52.2\% (RDR). Thus the genes follow the general trends of Phytophthora genes, where only onethird possess an intron (average length of 79 bases), of which the average is 1.5 introns per gene, and transcribed sequences have a higher GC content of 58\% (Kamoun, 2003). The sequences surrounding the transcription start site, translation start site, and exon/intron boundaries also generally follow the trend seen in other Phytophthora genes (Supplementary Figure 2).

All but one of the commonly observed Dicer protein domains are present in the $P$. sojae homologs, although only the two RNase III domains are present in both DCL1 and DCL2. DCL1 additionally contains the DEAD-box helicase and dsRBD domains, whereas the PAZ and dsrm domains are found only within DCL2, although the PAZ domain is less well conserved and harder to predict. In comparison, Pfam predicted domains in the DCL homologs from plants harbor almost all common domains in each homolog. This suggests the $P$. sojae homologs may have a differentiation of function in the two DCL loci more diverged than among the plant homologs. For all phylogenetic analyses presented, Pfam predicted domain structures are presented for each homolog included in the analysis to allow for comparison of domain structure along with phylogenetic placement.

\section{Gene Expression of DCL1, DCL2, and RDR}

To ascertain if $P$. sojae DCL1, DCL2, and RDR are functional loci and expressed in various life stages, gene expression was assessed

FIGURE 1 I Organization of $P$. sojae small RNA biogenesis genes and proteins, dicer-like (DCL) and RNA-dependent RNA polymerase (RDR). Genes are
labeled followed by their Gene ID from the EuMicrobeDB Database (eumicrobedb.org). In the genomic DNA diagrams, exons and introns are represented as black
bars and lines, respectively. $5^{\prime}$ and $3^{\prime}$ UTRs are represented as gray bars. Conserved domains are indicated by colored bars in the mRNA diagrams: dsrm, dsRNA
binding motif; RdRP, RNA-dependent RNA polymerase domain; PAZ, PAZ domain named for the proteins Piwi Argonaute and Zwille.


with qRT-PCR analysis. All three genes were actively transcribed in all examined tissues at a level 15- to 150-fold lower than the control genes $\beta$-Tubulin (Ps109498) and WS41 (Ps137777; protein of the BAR-domain family), and only small changes in relative expression levels were detected (Supplementary Figure 3 ). The only life stage samples that were significantly different than mycelium were zoospores (2.2 to 3.6-fold elevation in transcript levels) and DCL1 expression in germinated cysts (6.8fold decrease in transcript levels; $1.9-2.9$ decrease in DCL2 and RDR transcript levels was not significantly different than mycelium). It is unknown if these changes in expression would have a significant impact on small RNA levels.

\section{$P$. sojae DCL1 and DCL2 are Nucleus Localized}

Based on nuclear localization signal (NLS) prediction using pSORT II (Nakai and Horton, 1999), there were a few candidate NLS found in $P$. sojae DCL1, while no NLS were predicted in DCL2. NLS have not been defined functionally in P. sojae, and several classical NLS worked poorly in P. sojae (Fang and Tyler, 2016). To determine the subcellular localization of P. sojae DCL1 and DCL2 directly, the two full-length genes were amplified and fused at the $\mathrm{C}$ termini with green fluorescent protein (GFP). The fusion genes were then introduced into $P$. sojae transformants. Examination of three independent $P$. sojae transformants overexpressing DCL1-GFP or DCL2-GFP by confocal microscopy revealed that both Dicers showed strong nuclear localization (Figure 2, Supplementary Figure 4).

\section{Phylogenetic Analyses of the RNase III Domains}

A broad spectrum of eukaryotic species was selected to analyze the evolutionary origins of oomycete DCR homologs. In all, 72 DCR homologs were identified in 33 species, as listed in Supplementary Table 2A. Research in different organisms resulted in different naming conventions (DCR and DCL); when already annotated we retained the provided name, otherwise we used the DCR designation. The number of homologs present from each analyzed species varied. Plants had three to five DCL homologs, in contrast to most animals, which harbored one

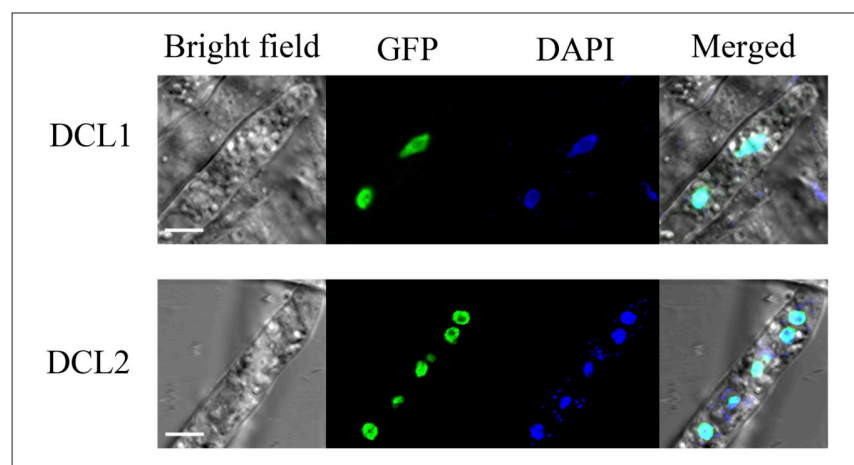

FIGURE 2 | Nuclear localization of DCL1 and DCL2 in P. sojae hyphae. Subcellular localization of both GFP tagged dicers overlaps with the nuclear marker DAPI. Scale bar, $5 \mu \mathrm{m}$. to two Dicers and one Drosha. Chromalveolates varied greatly between subgroups, for example oomycetes contained two DCL homologs and ciliates contained varied numbers of homologs. Two separate analyses were performed to elucidate the origins of the oomycete DCR homologs. In the first analysis (Figure 3, Supplementary Data Sheet 3), the amino acid sequences from the RNase IIIa and RNase IIIb domains of the DCR and Drosha homologs were concatenated to study the overall evolution of the key catalytic domains. The RNase III domains were selected rather than the full length protein sequences due to the great diversity of species included in the analysis. As expected, Drosha homologs formed an outgroup to DCR homologs. The two families of oomycete DCR homologs formed two distinct wellseparated clades. The DCL1 clade was associated with a poorly separated set of clades that included ciliates, higher plants, animals and fungi. The DCL2 homologs formed a much more basal clade with some affinity to green and brown algal DCL's.

To further investigate the basal placement of DCL2, a second analysis (Figure 4, Supplementary Figures 5A,B, Supplementary Data Sheet 4) was performed comparing the RNase IIIa and RNase IIIb (indicated in the Supplementary Figures 5A,B, respectively) domains of all DCR and Drosha homologs individually to enable more detailed analysis of this domains' evolution. Six additional sequences from bacterial and yeast ribonucleases, which only contain a single RNase III domain, were included to provide more diversity in the tree, especially at basal levels. The RNase IIIa domains of oomycete DCL1 homologs clustered with higher plant RNase IIIa within the DCR A clade, while the RNase IIIb domains clustered with ciliate and animal homologs within the DCR B clade.

The analysis of the RNase III domains of the oomycete DCL2 homologs revealed that the two domains formed distinct clusters within a poorly resolved set of basal clades that includes two Drosha clades. The DCL2 RNase IIIa domains showed weak affinity with some diatom, excavate and yeast sequences, while the RNase IIIb domains showed weak affinity with bacterial Rnc sequences. The RNase III domain from the budding yeast DCR (SaccDCR), which has recently been described as a noncanonical Dicer that may function as a homodimer in an endogenous siRNA pathway (Drinnenberg et al., 2009), clusters tightly with the oomycete DCL2 RNase IIIa domain. Therefore the four RNase III domains of the oomycete DCRs all show widely different phylogenetic affinities.

While all the oomycetes tested, from Phytophthora to Saprolegnia, shared a common set of DCR homologs with oomycete-specific origins, the other Stramenopiles tested only had a single DCR homolog that had a different origin. For the brown algae Ectocarpus siliculosus, both the analysis of the combined RNase III domains and the separated domains showed the sequences clustering with homologs from green algae. For the diatoms $P$. tricornutum and T. pseudonana, the combined analysis placed the DCR homologs within a poorly resolved set of clades including excavates, microsporidia, and fungi (Supplementary Figure 5). The separated analysis showed unexpectedly that the RNase IIIa domains of the two diatom DCRs showed very different affinities. The T. pseudonana sequence clustered with the excavates and the oomycetes, 

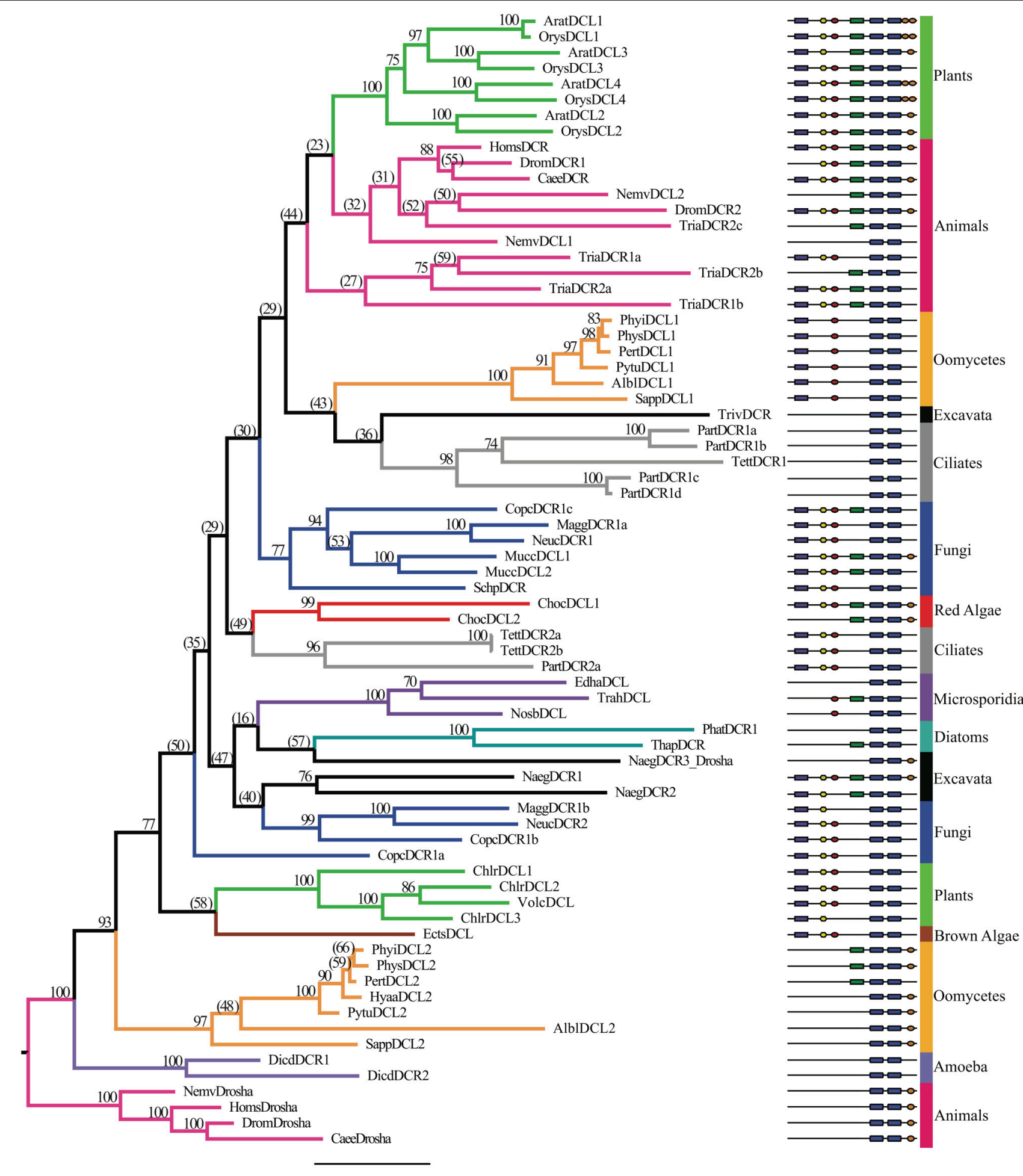

0.3

FIGURE 3 | Phylogenetic tree of Dicer homologs based on the concatenated RNase IIla and RNase IIlb domains. Bayesian support values are shown next to nodes. Branches are colored to denote major species groups, as labeled on the right. Gene model diagrams (not drawn to scale) indicate Pfam predicted domains: left to right: purple rectangle, DEAD-box helicase; yellow hexagon, helicase-C domain; red oval, dsRBD; green rectangle, PAZ domain; blue rounded boxes, RNase IIla and RNase Illb domains; orange oval, dsrm. Species abbreviations are defined in Supplementary Table 2A. 


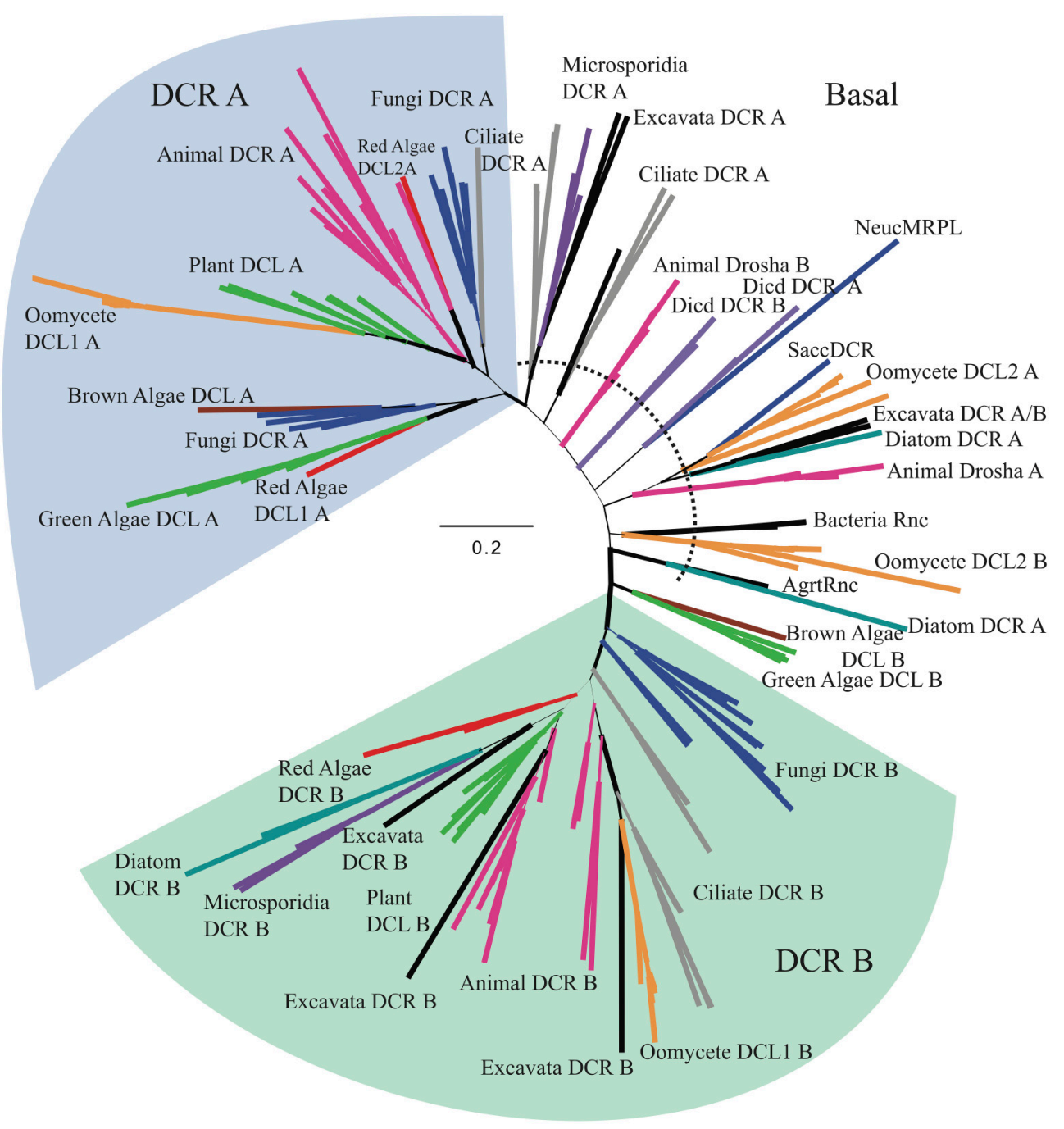

FIGURE 4 | Radial, phylogenetic tree of Dicer homologs based on individual RNase Illa and RNase Illb domains. Branch thicknesses signify significance of Bayesian support. Branches are colored to denote major species groups, as in Figures 2, 4, and $\mathbf{5}$. Species abbreviations are defined in Supplementary Table 2A.

while the $P$. tricornutum clustered with a bacterial Rnc (from Agrobacterium tumefaciens). On the other hand, the two RNase IIIb domains clustered tightly together, with affinity to microsporidial sequences.

\section{Phylogenetic Analysis of the DEAD-box Helicase Domain}

Analysis of conserved domain structures in oomycete small RNA-processing proteins confirmed the presence of a putative DEAD-box helicase domain in the DCL1 homologs, but not within the DCL2 homologs (e.g., Figure 1). The amino terminus of the RDR homolog also contained a DEAD-box helicase domain, together with a related helicase- $\mathrm{C}$ domain. This domain arrangement has been reported previously in Dictyostelium discoideum (Martens et al., 2002; Kuhlmann et al., 2005; Hinas et al., 2007), but not in other organisms. In addition to the oomycete and $D$. discoideum RDR homologs, we identified the same DEAD-box helicase-helicase-C-RDRP domain structure in RDR homologs from the lower animals Hydra magnipapillata and Nematostella vectensis. As reported in D. discoideum (Martens et al., 2002) and as determined bioinformatically for $H$. magnipapillata and $N$. vectensis, the altered RDR structure is coincidental with loss of DEAD-box helicase domains in each organisms' DCR homologs.

DEAD-box helicase sequences from both DCR and RDR homologs were analyzed in a single tree to elucidate the potential origins of the oomycete sequences. Figure 5 (Supplementary Data Sheet 5) displays the phylogenetic analysis of the DEADbox helicase domains. The DEAD-box helicases from RDR homologs showed a strong separation from the DEADbox helicase domains from the DCR homologs, indicating distinct evolutionary origins. The DCR clade contained a large number of poorly resolved subclades corresponding to 


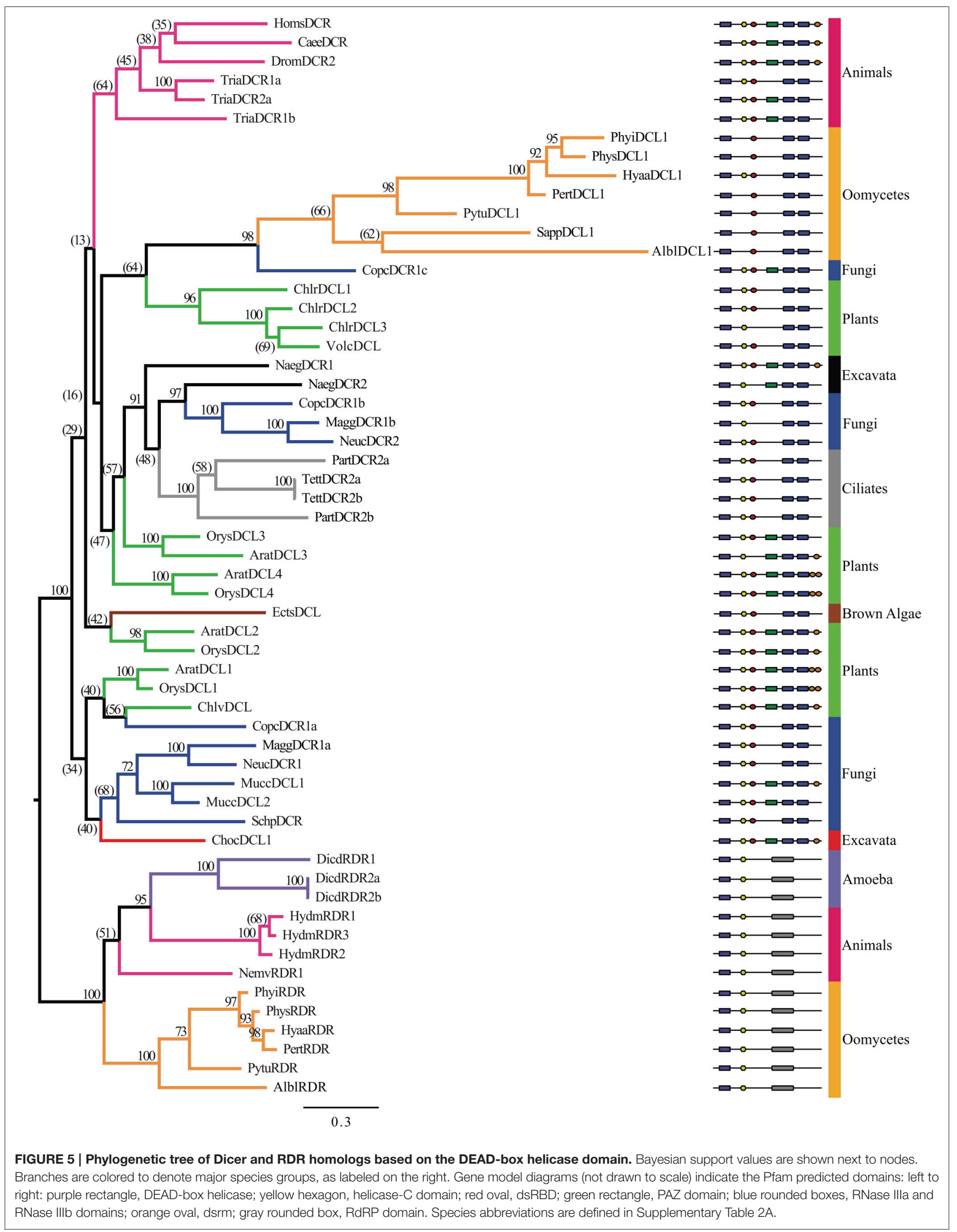


different kingdoms. The oomycete DCL1 sub-tree showed exceptionally long branch lengths, suggesting rapid divergence of the sequences within the oomycetes. Interestingly, the RDR DEAD-box helicase domains from oomycetes, Dictyostelium, Hydra, and Nematostella clustered together and were strongly separated from all the DCL1 dead box domains.

A reciprocal best-blast analysis was performed to determine if there were potential orthology relationships between the DEAD-box helicase-containing RDR homologs. The DEAD-box helicase domains from $D$. discoideum and $H$. magnipapillata RDR homologs were reciprocal best-blast hits, suggesting a common evolutionary origin at the base of the animal and amoebozoa kingdoms. However, the DEAD-box helicase domains from the oomycete RDR homologs showed no consistent affinity with other evolutionary groups, including animal and amoebozoa RDR homologs or with the oomycete DCL1 homologs.

\section{Phylogenetic Analysis of the RDRP Domain from RDR Homologs}

To refine the origin of oomycete RDR homologs as examined by Zong et al. (2009), we included additional oomycete, Stramenopile, protist, and basal metazoan sequences to broaden the phylogenetic analysis. In all, 90 RDR homologs were identified in 38 species, as listed in Supplementary Table 2B. Similar to DCR homologs, the number of homologs present for each species analyzed varied. Plants had five to six RDR homologs, whereas most animals did not have RDR homologs at all. A small number of lower animals, including Ixodes scapularis, Branchiostoma floridae, nematodes, and cnidarians contained multiple RDR homologs, ranging from 3 to 7. Stramenopiles varied greatly between subgroups. For example, oomycetes from Phytophthora to Albugo contained only one RDR homolog, but Saprolegnia contained up to five homologs, and diatoms contained a varied numbers of homologs. Phylogenetic analysis of the RDRP domain (Figure 6, Supplementary Data Sheet 6) confirmed the overall tree published by Zong et al. (2009) with some changes. In the previous analysis, the oomycete homolog (P. ramorum) was basal to the $\mathrm{RDR} \alpha$ animal clade. The current study refined the placement of Phytophthora, Hyaloperonospora, Peronospora, Pythium, and Albugo homologs as basal to the $\mathrm{RDR} \gamma$ clade along with Hydra homologs, while the Saprolegnia and Ectocarpus homologs clustered with fungi in the RDR $\alpha$ clade (Figure 6, bottom). The occupants of the RDR $\beta$ clade were also clarified, with ciliate homologs basal to the clade. Some fungal and animal sequences were located in the $\operatorname{RDR} \gamma$ cluster, but others did not associate clearly with either clade $\beta$ or clade $\gamma$. The larger number of generations in the current analysis (32 million versus 1 million) allowed better refinement of the overall tree, with each major RDR group showing fairly good support for a tree with this diverse range of species. The separation of primarily two main clades with a high Bayesian posterior probability of 100 only partially supports the previous study's hypothesis that there were three RDR homologs in the eukaryotic ancestor (Zong et al., 2009). In our analysis, the Bayesian posterior probability of separation of the $\beta$ and $\gamma$ clades was only $29 \%$.

\section{Conservation Analysis of Key Residues}

To analyze the potential for the oomycete DCR and RDR homologs to possess their canonical functions, conserved residues shown to be important for catalytic activity were compared within the domain alignments (Supplementary Figures 6-8). For the RNase III domain (Supplementary Figure 6), catalytic residues ExxGD and DxxE were found at the beginning and end of the domain, respectively (Lepère et al., 2009). For the RDRP domain (Supplementary Figure 7) the key residues are DLDGD, with the last aspartic acid required for catalytic activity (Lee and Collins, 2007; Marker et al., 2010). Manual analysis of the key catalytic residues in the RNase III and RDRP domains of the oomycete DCR and RDR homologs showed perfect conservation of the consensus sequence for the majority of the homologs. Variations in consensus sequence in the RNase III domain included: all four Drosha RNase IIIa homologs had NxxE in the second motif, T. adhaerens DCL1a RNase IIIa, Naegleria gruberi DCR3 RNase IIIa, P. tricornutum DCR1 RNase IIIa, and Albugo laibachii DCL2 RNase IIIb had variation at one or both residues of the second motif, Naegleria gruberi DCR3 RNase IIIb had KxxxS in the first motif, and T. adhaerens DCL1b RNase IIIa, T. adhaerens DCL2b RNase IIIb, P. tricornutum DCR1 RNase IIIb, T. pseudonana DCR RNase IIIb, Albugo laibachii DCL2 RNase IIIa, and Neurospora crassa MRPL3 had variations in both motifs. Variations in the consensus sequence in the RDRP domain were mostly limited to the second residue (making it DxDGD). Only one homolog with a complete sequence covering that region had more than the change at the second residueMucor circinelloides RDR2 (DPDED). In the case of Albugo laibachii RDR (-DSE), the sequence is not clear at that site due to unresolved intron placement. More significant deviations from consensus sequences were observed, however, in the DEAD-box helicase domain (Supplementary Figure 8), especially in the oomycete DCL1 homologs. Whereas oomycete RDR homologs show conservation of the key site residues GxGKT (ATP-binding residues) and $\mathrm{DE}[\mathrm{C} / \mathrm{A}] \mathrm{H}\left(\mathrm{Mg}^{2+}\right.$-binding residues) observed in canonical DEAD-box helicase domains from DCR homologs, the oomycete DCL1 homologs had numerous substitutions in both sequences. Another commonly observed variation was DEVH at the second motif, seen in Dictyostelium RDR homologs and Chondrus crispus DCL1. Supplementary Figure 9 compares the sequences of the key motifs of the DEAD-box helicase domains among different Stramenopile homologs.

\section{DISCUSSION}

In contrast to other housekeeping genes, the genes encoding the small RNA machinery of eukaryotes display a remarkable degree of diversity, not only in primary sequences, but also in terms of domain structure, and gene family size. Currently the evolutionary forces that drive this diversity, and the mechanisms by which the diversity has been generated remain unknown. While the small RNA machinery of plants, animals and fungi has been quite well characterized, the small RNA machinery of the Stramenopile kingdom, which includes both photosynthetic 


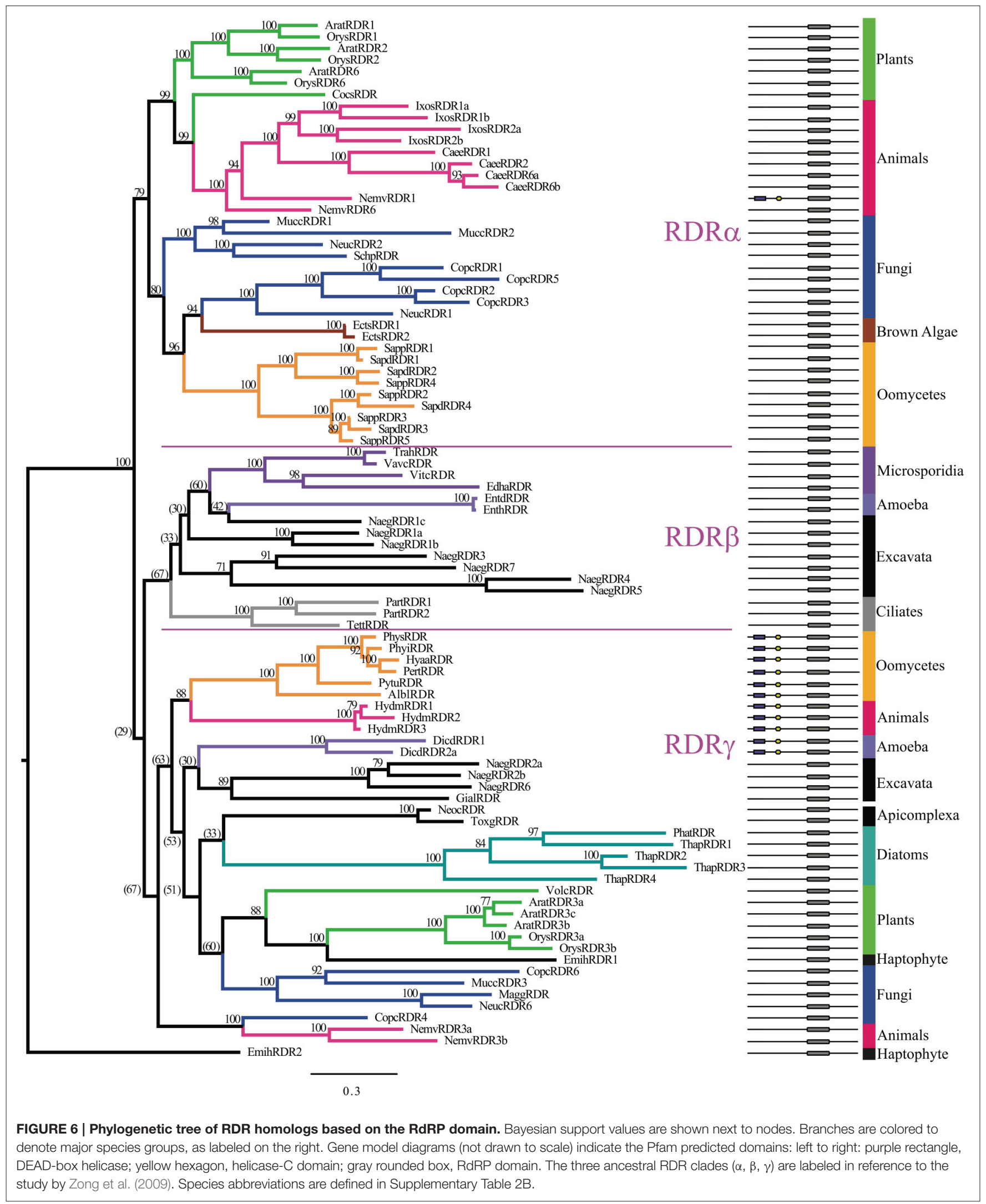


algae and destructive oomycete pathogens, has received little attention. Here, beginning with the validation of gene models using amplified cDNAs from $P$. sojae, we have demonstrated that the DCL and RDR proteins of oomycetes display unusually distinctive evolutionary origins.

In contrast to other species, such as plants, whose multiple DCR homologs are the result of gene duplication after splitting of the major group lineages, the two DCR homologs in oomycetes have widely divergent origins. Oomycete DCL1 homologs are part of a poorly resolved clade including ciliates, plants, animals, and fungi. This group is well separated from oomycete DCL2 homologs, which resolved more basally in the tree along with Dictyostelium DCR and animal Drosha homologs, although these three groups are well separated from each other. This pattern appears in both RNase III domain analyses. The oomycete DCL1 RNase IIIa domain is more plant-like, while the DCL1 RNase IIIb is more similar to ciliates and basal animals. The DCL2 RNase III domains are both more basal. The DCL2 Rnase IIIa domain is similar to yeast, excavate, and diatom RNase domains and very weakly to Drosha RNase IIIa domains while the RNAse IIIb domain is more similar to bacterial Rnc domains. The structural differences between oomycete DCL2 and animal Drosha homologs provide further support for a different function for these proteins. Although both DCR and Drosha homologs contain two RNaseIII domains, a hallmark of Drosha has been the lack of a PAZ domain, which is critical for proper DCR processing of small RNA duplexes. In contrast, the oomycete DCL2 protein has a conserved PAZ domain, suggesting its function to be more DCR-like than Drosha-like. We hypothesize that the functions of DCL1 and DCL2 have independent evolutionary origins and thus must have different functions given the differences in domain content (Figure 1). This is in line with the previously observed support for two distinct small RNA classes of predominantly 21or 25-nt in length (Fahlgren et al., 2013) that might be processed each by one of the two DCL proteins.

The localization of both oomycete DCL1 and DCL2 homologs to the nucleus does not help to resolve their function. For comparison, A. thaliana DCL1 and DCL2, which are responsible for miRNA and viral siRNA pathways, respectively, have predicted NLS sequences, and DCL3 and DCL4, which are responsible for endogenous siRNA and tasiRNA pathways, respectively, do not have predicted NLS sequences. The Drosha homolog from $H$. sapiens, which processes pri-miRNA in the nucleus, has a predicted NLS sequence, but the DCR homolog does not. In other organisms, miRNA processing usually occurs in the nucleus and siRNA processing usually occurs in the cytoplasm, yet in Phytophthora it appears that the 21- and 25-nt processing pathways both must occur in the nucleus. The small specks observed in our images may be evidence of cajal bodies in Phytophthora nuclei, which could be the centers of miRNA- and siRNA-processing. We cannot rule out the possibility of partial or temporary localization of either DCL in the cytoplasm, as the signal would be diluted and not readily detectable. However our images support predominant nuclear localization of both DCLs.

The origin of the DEAD-box helicase domain in oomycete RDR homologs is not clear. RDR homologs with DEADbox helicase domains were relatively uncommon among the eukaryote genomes examined. Among the oomycetes, only species in the class Peronosporomycetidae (Phytophthora, Pythium, downy mildew, Albugo) had this arrangement; those from S. parasitica (Saprolegniomycetidae) did not. Among other eukaryotes, only RDRs from Dictyostelium (Amebozoa), and from Hydra and Nematostella (Animalia) had this arrangement. The DEAD box domains from all of the RDR homologs, including the peronosporomycetes, formed a single clade separated with $100 \%$ support from the DEAD box domains of the Dicer homologs. This observation suggests that the RDR-associated DEAD box domains have an ancient common origin that is distinct from the Dicer DEAD box domains. This inference is supported in part by the observation that the peronosporomycete and Hydra RDRs also form a cluster within the RDR $\gamma$ subgroup based on the RDRP domains of the proteins. The Dictyostelium and Nematostella RDRs also fall within the RDR $\gamma$ subgroup, but do not cluster unambiguously with the peronosporomycete and Hydra RDRs. If the DEAD box domaincontaining RDRs all have a common origin, then either the DEAD box domains must have been lost from some RDRs (e.g., in the RDR $\gamma$ subgroup) or the DEAD box-containing RDRs may have been entirely lost from some eukaryotic lineages (e.g., those containing no $\gamma$ subgroup RDRs).

The presence of DEAD box domains in the peronosporomycete, Dictyostelium and Nematostella RDRs coincides with the loss of DEAD box domains from a subset of the Dicer homologs encoded in each of those genomes (DCL2's in the case of the peronosporomycetes). However, the significance of this coincidence is not clear as in Saprolegnia DEAD box domains are absent from its DCL2 as well as all its RDRs. Furthermore, many other eukaryotes that contain Dicer homologs lacking a DEAD box domain also lack RDRs with DEAD box domains.

Of the organisms that contain a DEAD-box helicase domain in the RDR homolog, peronosporomycetes are unique in that they also have a DEAD-box helicase domain in one of the Dicer homologs (i.e., in their DCL1's); Dictyostelium, Hydra (not shown), and Nematostella only have Dicer homologs that lack DEAD box domains. Furthermore the branch lengths of the DEAD box domains of the peronosporomycete DCL1's are unusually long, whereas the branch lengths of the DCL1 RNase III domains are not exceptionally long. Whether this indicates the DCL1 DEAD box domains are currently degenerating or evolving a novel function is unclear. The oomycete DCL1 homologs are also unique in that they are the only DEADbox domain-containing proteins that lack a predicted HelicaseC domain. Although the oomycete DCL1 sequences are highly divergent from other DCR homologs, it is unclear what the effect of these changes would be on the catalytic activity of the domain. In the $\mathrm{DE}[\mathrm{C} / \mathrm{A}] \mathrm{H}$ box sequence, for example, switching the aspartic acid and glutamic acid residues may not have a huge impact on function, and a tryptophan in the third, most variable, position has unknown significance. As well, even though the other DEAD-box helicase domains in the analysis have a histidine in the fourth position, other members of the helicase family contain aspartic acid in the fourth position. Therefore the oomycete DCL1 homologs could potentially retain 
their function. Without biochemical or mutagenesis studies it is unclear whether the divergence observed for the DEAD-box helicase is related to function of the domain.

In D. discoideum, Martens et al. (2002) suggested that the alternative DEAD box domain arrangement indicated that cleavage of dsRNA and amplification of the signal and guide RNAs are spatially linked, and therefore that the placement of the domain was not critical to function. A study of the DEADbox helicase from $H$. sapiens DCR suggested the domain aided in the processing of thermodynamically unstable dsRNA structures, such as miRNA precursors, and restricted the processivity of DCR on perfectly-matched substrates (Soifer et al., 2008). It is not clear though how these activities would facilitate RDR function. In $H$. sapiens there is no RDR homolog at all, so the DEADbox helicase may serve a different role in species that possess RDR homologs. In Dictyostelium, the DEAD-box helicasecontaining RDR homolog is implicated in the production of $\sim 23$ nt siRNAs (Martens et al., 2002). In our phylogenetic analyses, both DCL2 and RDR from P. sojae and Dictyostelium clustered together, suggesting their similarity of domain structure might also indicate a similarity of function. We speculate that oomycete DCL2 could be involved in a nuclear-localized siRNA/25-nt small RNA pathway.

In the analysis of the DCR A subgroup of RNase III sequences, the oomycete sequences clustered significantly more tightly with plant and animal sequences, and more distantly from fungal sequences, despite the closer evolutionary relationship between animals and fungi. We note that plants, animals and oomycetes (Fahlgren et al., 2013) have miRNA pathways, whereas canonical miRNAs have yet to be discovered in fungi. Thus we speculate that in oomycetes DCL1 could be involved in the miRNA/21nt small RNA pathway. This is supported by evidence from Vetukuri et al. (2012), who showed that partial silencing of DCL1 in $P$. infestans led to decreased production of assayed 21-nt small RNAs.

Work is currently underway to analyze the small RNA landscape in $P$. sojae through high-throughput sequencing of small RNA from various life stages and infection time points. Ultimately, a better understanding of oomycete small RNA

\section{REFERENCES}

Ah-Fong, A. M. V., Bormann-Chung, C. A., and Judelson, H. S. (2008). Optimization of transgene-mediated silencing in Phytophthora infestans and its association with small-interfering RNAs. Fungal Genet. Biol. 45, 1197-1205. doi: 10.1016/j.fgb.2008.05.009

Alemdehy, M. F., Haanstra, J. R., de Looper, H. W. J., van Strien, P. M. H., Verhagen-Oldenampsen, J., Caljouw, Y., et al. (2015). ICL-induced miR139$3 p$ and miR199a-3p have opposite roles in hematopoietic cell expansion and leukemic transformation. Blood 125, 3937-3948. doi: 10.1182/blood-2014-11612507

Aravin, A. A., Naumova, N. M., Tulin, A. V., Vagin, V. V., Rozovsky, Y. M., and Gvozdev, V. A. (2001). Double-stranded RNA-mediated silencing of genomic tandem repeats and transposable elements in the D. melanogaster germline. Curr. Biol. 11, 1017-1027. doi: 10.1016/S0960-9822(01)00299-8

Baurain, D., Brinkmann, H., Petersen, J., Rodríguez-Ezpeleta, N., Stechmann, A., Demoulin, V., et al. (2010). Phylogenomic evidence for separate acquisition of pathways may lead to improved techniques for silencing genes of interest or discovery of small RNA pathways related to hostpathogen interactions.

\section{AUTHOR CONTRIBUTIONS}

SB, YF, CP, BT, and NG all contributed to designing and analyzing experiments, specifically SB was the primary contributor with help from CP and NG on gene expression design and NG and BT on phylogenetic and conservation analysis; YF designed and performed the localization experiments with guidance from BT. All authors also contributed to the drafting, revising, and final approval of the manuscript and are accountable for its accuracy.

\section{FUNDING}

This work was supported by the USDA National Research Initiative (grant number 2008-35600-18780); and the USDA Agricultural Research Service (CRIS number 5358-2200003900D).

\section{ACKNOWLEDGMENTS}

We thank James C. Carrington and in particular Noah Fahlgren for advice on silencing genetics, Christopher M. Sullivan for excellent technical support, and the Center for Genome Research and Biocomputing, Oregon State University for use of the bioinformatics computer cluster and for DNA sequencing. Mention of trade names or commercial products in this manuscript are solely for the purpose of providing specific information and do not imply recommendation or endorsement by the U.S. Department of Agriculture. We declare that the authors have no competing interests.

\section{SUPPLEMENTARY MATERIAL}

The Supplementary Material for this article can be found online at: http://journal.frontiersin.org/article/10.3389/fpls.2016. 00284

plastids in cryptophytes, haptophytes, and stramenopiles. Mol. Biol. Evol. 27, 1698-1709. doi: 10.1093/molbev/msq059

Baxter, L., Tripathy, S., Ishaque, N., Boot, N., Cabral, A., Kemen, E., et al. (2010). Signatures of adaptation to obligate biotrophy in the Hyaloperonospora arabidopsidis genome. Science 330, 1549-1551. doi: 10.1126/science.1195203

Bernstein, E., Caudy, A. A., Hammond, S. M., and Hannon, G. J. (2001). Role for a bidentate ribonuclease in the initiation step of RNA interference. Nature 409, 363-366. doi: 10.1038/35053110

Blanco, F. A., and Judelson, H. S. (2005). A bZIP transcription factor from Phytophthora interacts with a protein kinase and is required for zoospore motility and plant infection. Mol. Microbiol. 56, 638-648. doi: 10.1111/j.13652958.2005.04575.x

Braun, L., Cannella, D., Ortet, P., Barakat, M., Sautel, C. F., Kieffer, S., et al. (2010). A complex small RNA repertoire is generated by a plant/fungallike machinery and effected by a metazoan-like Argonaute in the singlecell human parasite Toxoplasma gondii. PLoS Pathog. 6:e1000920. doi: 10.1371/journal.ppat.1000920 
Burge, C., and Karlin, S. (1997). Prediction of complete gene structures in human genomic DNA. J. Mol. Biol. 268, 78-94. doi: 10.1006/jmbi.1997.0951

Cerutti, H., and Casas-Mollano, J. A. (2006). On the origin and functions of RNA-mediated silencing: from protists to man. Curr. Genet. 50, 81-99. doi: 10.1007/s00294-006-0078-x

Dalmay, T., Hamilton, A., Rudd, S., Angell, S., and Baulcombe, D. C. (2000). An RNA-dependent RNA polymerase gene in Arabidopsis is required for posttranscriptional gene silencing mediated by a transgene but not by a virus. Cell 101, 543-553. doi: 10.1016/S0092-8674(00)80864-8

de Jong, D., Eitel, M., Jakob, W., Osigus, H.-J., Hadrys, H., Desalle, R., et al. (2009). Multiple dicer genes in the early-diverging metazoa. Mol. Biol. Evol. 26, 1333-1340. doi: 10.1093/molbev/msp042

Denli, A. M., and Hannon, G. J. (2003). RNAi: an ever-growing puzzle. Trends Biochem. Sci. 28, 196-201. doi: 10.1016/S0968-0004(03)00058-6

Derevnina, L., Chin-Wo-Reyes, S., Martin, F., Wood, K., Froenicke, L., Spring, O., et al. (2015). Genome sequence and architecture of the tobacco downy mildew pathogen, Peronospora tabacina. Mol. Plant Microbe Interact. 28, 1198-1215. doi: 10.1094/MPMI-05-15-0112-R

Dlakić, M. (2006). DUF283 domain of Dicer proteins has a double-stranded RNAbinding fold. Bioinformatics 22, 2711-2714. doi: 10.1093/bioinformatics/btl468

Drinnenberg, I. A., Weinberg, D. E., Xie, K. T., Mower, J. P., Wolfe, K. H., Fink, G. R., et al. (2009). RNAi in budding yeast. Science 326, 544-550. doi: $10.1126 /$ science. 1176945

Dunoyer, P., Himber, C., and Voinnet, O. (2005). DICER-LIKE 4 is required for RNA interference and produces the 21-nucleotide small interfering RNA component of the plant cell-to-cell silencing signal. Nat. Genet. 37, 1356-1360. doi: $10.1038 / \mathrm{ng} 1675$

Fahlgren, N., Bollmann, S. R., Kasschau, K. D., Cuperus, J. T., Press, C. M., Sullivan, C. M., et al. (2013). Phytophthora have distinct endogenous small RNA populations that include short interfering and microRNAs. PLOS ONE 8:e77181. doi: 10.1371/journal.pone.0077181

Fang, Y., and Tyler, B. M. (2016). Efficient disruption and replacement of an effector gene in the oomycete Phytophthora sojae using CRISPR/Cas9. Mol. Plant Pathol. 17, 127-139. doi: 10.1111/mpp.12318

Finn, R. D., Mistry, J., Tate, J., Coggill, P., Heger, A., Pollington, J. E., et al. (2010). The Pfam protein families database. Nucleic Acids Res. 38, D211-D222. doi: 10.1093/nar/gkp985

Fry, W. (2008). Phytophthora infestans: the plant (and R gene) destroyer. Mol. Plant Pathol. 9, 385-402. doi: 10.1111/j.1364-3703.2007.00465.x

Gasciolli, V., Mallory, A. C., Bartel, D. P., and Vaucheret, H. (2005). Partially redundant functions of Arabidopsis DICER-like enzymes and a role for DCL4 in producing trans-acting siRNAs. Curr. Biol. 15, 1494-1500. doi: 10.1016/j.cub.2005.07.024

Gaulin, E., Jauneau, A., Villalba, F., Rickauer, M., Esquerré-Tugayé, M.-T., and Bottin, A. (2002). The CBEL glycoprotein of Phytophthora parasitica varnicotianae is involved in cell wall deposition and adhesion to cellulosic substrates. J. Cell Sci. 115, 4565-4575. doi: 10.1242/jcs.00138

Ghildiyal, M., and Zamore, P. D. (2009). Small silencing RNAs: an expanding universe. Nat. Rev. 10, 94-108. doi: 10.1038/nrg2504

Grünwald, N. J., Goss, E. M., and Press, C. M. (2008). Phytophthora ramorum: a pathogen with a remarkably wide host range causing sudden oak death on oaks and ramorum blight on woody ornamentals. Mol. Plant Pathol. 9, 729-740. doi: 10.1111/j.1364-3703.2008.00500.x

Haas, B. J., Kamoun, S., Zody, M. C., Jiang, R. H. Y., Handsaker, R. E., Cano, L. M., et al. (2009). Genome sequence and analysis of the Irish potato famine pathogen Phytophthora infestans. Nature 461, 393-398. doi: 10.1038/nature08358

Hamilton, A. J., and Baulcombe, D. C. (1999). A species of small antisense RNA in posttranscriptional gene silencing in plants. Science 286, 950-952. doi: 10.1126/science.286.5441.950

Hammond, S. M., Bernstein, E., Beach, D., and Hannon, G. J. (2000). An RNAdirected nuclease mediates post-transcriptional gene silencing in Drosophila cells. Nature 404, 293-296. doi: 10.1038/35005107

Hardham, A. R. (2001). "Investigations of oomycete cell biology," in Molecular and Cell Biology of Filamentous Fungi, ed N. Talbot (Oxford: Oxford University Press), 127-155.

Herr, A. J., Jensen, M. B., Dalmay, T., and Baulcombe, D. C. (2005). RNA polymerase IV directs silencing of endogenous DNA. Science 308, 118-120. doi: $10.1126 /$ science. 1106910
Hinas, A., Reimegård, J., Wagner, E. G. H., Nellen, W., Ambros, V. R., and Söderbom, F. (2007). The small RNA repertoire of Dictyostelium discoideum and its regulation by components of the RNAi pathway. Nucleic Acids Res. 35, 6714-6726. doi: 10.1093/nar/gkm707

Huang, A., He, L., and Wang, G. (2011). Identification and characterization of microRNAs from Phaeodactylum tricornutum by high-throughput sequencing and bioinformatics analysis. BMC Genomics 12:337. doi: 10.1186/1471-2164$12-337$

Huelsenbeck, J. P., and Ronquist, F. (2001). MRBAYES: Bayesian inference of phylogenetic trees. Bioinformatics 17, 754-755. doi: 10.1093/bioinformatics/17.8.754

Jiang, R. H. Y., de Bruijn, I., Haas, B. J., Belmonte, R., Löbach, L., Christie, J., et al. (2013). Distinctive expansion of potential virulence genes in the genome of the oomycete fish pathogen Saprolegnia parasitica. PLoS Genet. 9:e1003272. doi: 10.1371/journal.pgen.1003272

Judelson, H. S., and Tani, S. (2007). Transgene-induced silencing of the zoosporogenesis-specific NIFC gene cluster of Phytophthora infestans involves chromatin alterations. Eukaryotic Cell 6, 1200-1209. doi: 10.1128/EC. 00311-06

Judelson, H. S., and Whittaker, S. L. (1995). Inactivation of transgenes in Phytophthora infestans is not associated with their deletion, methylation, or mutation. Curr. Genet. 28, 571-579. doi: 10.1007/BF00518171

Kamoun, S. (2003). Molecular genetics of pathogenic oomycetes. Eukaryotic Cell 2, 191-199. doi: 10.1128/EC.2.2.191-199.2003

Kasschau, K. D., Fahlgren, N., Chapman, E. J., Sullivan, C. M., Cumbie, J. S., Givan, S. A., et al. (2007). Genome-wide profiling and analysis of Arabidopsis siRNAs. PLoS Biol. 5:e57. doi: 10.1371/journal.pbio.0050057

Kearse, M., Moir, R., Wilson, A., Stones-Havas, S., Cheung, M., Sturrock, S., et al. (2012). Geneious basic: an integrated and extendable desktop software platform for the organization and analysis of sequence data. Bioinformatics 28 , 1647-1649. doi: 10.1093/bioinformatics/bts199

Kuhlmann, M., Borisova, B. E., Kaller, M., Larsson, P., Stach, D., Na, J., et al. (2005). Silencing of retrotransposons in Dictyostelium by DNA methylation and RNAi. Nucleic Acids Res. 33, 6405-6417. doi: 10.1093/nar/gki952

Latijnhouwers, M., and Govers, F. (2003). A Phytophthora infestans G-protein beta subunit is involved in sporangium formation. Eukaryotic Cell 2, 971-977. doi: 10.1128/EC.2.5.971-977.2003

Lee, H.-C., Li, L., Gu, W., Xue, Z., Crosthwaite, S. K., Pertsemlidis, A., et al. (2010). Diverse pathways generate microRNA-like RNAs and Dicerindependent small interfering RNAs in fungi. Mol. Cell 38, 803-814. doi: 10.1016/j.molcel.2010.04.005

Lee, R. C., Feinbaum, R. L., and Ambros, V. (1993). The C. elegans heterochronic gene lin-4 encodes small RNAs with antisense complementarity to lin-14. Cell 75, 843-854. doi: 10.1016/0092-8674(93)90529-Y

Lee, S. R., and Collins, K. (2007). Physical and functional coupling of RNAdependent RNA polymerase and Dicer in the biogenesis of endogenous siRNAs. Nat. Struct. Mol. Biol. 14, 604-610. doi: 10.1038/nsmb1262

Lee, Y., Ahn, C., Han, J., Choi, H., Kim, J., Yim, J., et al. (2003). The nuclear RNase III Drosha initiates microRNA processing. Nature 425, 415-419. doi: $10.1038 /$ nature01957

Lepère, G., Nowacki, M., Serrano, V., Gout, J.-F., Guglielmi, G., Duharcourt, S., et al. (2009). Silencing-associated and meiosis-specific small RNA pathways in Paramecium tetraurelia. Nucleic Acids Res. 37, 903-915. doi: 10.1093/nar/gkn1018

Lévesque, C. A., Brouwer, H., Cano, L., Hamilton, J. P., Holt, C., Huitema, E., et al. (2010). Genome sequence of the necrotrophic plant pathogen Pythium ultimum reveals original pathogenicity mechanisms and effector repertoire. Genome Biol. 11:R73. doi: 10.1186/gb-2010-11-7-r73

Links, M. G., Holub, E., Jiang, R. H. Y., Sharpe, A. G., Hegedus, D., Beynon, E., et al. (2011). De novo sequence assembly of Albugo candida reveals a small genome relative to other biotrophic oomycetes. BMC Genomics 12:503. doi: 10.1186/1471-2164-12-503

Liu, Q., Feng, Y., and Zhu, Z. (2009). Dicer-like (DCL) proteins in plants. Funct. Integr. Genomics 9, 277-286. doi: 10.1007/s10142-0090111-5

MacRae, I. J., Zhou, K., Li, F., Repic, A., Brooks, A. N., Cande, W. Z., et al. (2006). Structural basis for double-stranded RNA processing by Dicer. Science 311, 195-198. doi: 10.1126/science.1121638 
Makeyev, E. V., and Bamford, D. H. (2002). Cellular RNA-dependent RNA polymerase involved in posttranscriptional gene silencing has two distinct activity modes. Mol. Cell 10, 1417-1427. doi: 10.1016/S1097-2765(02)00780-3

Marker, S., Le Mouël, A., Meyer, E., and Simon, M. (2010). Distinct RNAdependent RNA polymerases are required for RNAi triggered by doublestranded RNA versus truncated transgenes in Paramecium tetraurelia. Nucleic Acids Res. 38, 4092-4107. doi: 10.1093/nar/gkq131

Martens, H., Novotny, J., Oberstrass, J., Steck, T. L., Postlethwait, P., and Nellen, W. (2002). RNAi in Dictyostelium: the role of RNA-directed RNA polymerases and double-stranded RNase. Mol. Biol. Cell 13, 445-453. doi: 10.1091/mbc.0104-0211

Mehta, A., Mann, M., Zhao, J. L., Marinov, G. K., Majumdar, D., Garcia-Flores, Y., et al. (2015). The microRNA-212/132 cluster regulates B cell development by targeting Sox4. J. Exp. Med. 212, 1679-1692. doi: 10.1083/jcb.2107OIA191

Nakai, K., and Horton, P. (1999). PSORT: a program for detecting sorting signals in proteins and predicting their subcellular localization. Trends Biochem. Sci. 24, 34-36. doi: 10.1016/S0968-0004(98)01336-X

Norden-Krichmar, T. M., Allen, A. E., Gaasterland, T., and Hildebrand, M. (2011). Characterization of the small RNA transcriptome of the diatom, Thalassiosira pseudonana. PLoS ONE 6:e22870. doi: 10.1371/journal.pone.0022870

Park, W., Li, J., Song, R., Messing, J., and Chen, X. (2002). CARPEL FACTORY, a Dicer homolog, and HEN1, a novel protein, act in microRNA metabolism in Arabidopsis thaliana. Curr. Biol. 12, 1484-1495. doi: 10.1016/S09609822(02)01017-5

Pfaffl, M. W., Horgan, G. W., and Dempfle, L. (2002). Relative expression software tool (REST) for group-wise comparison and statistical analysis of relative expression results in real-time PCR. Nucleic Acids Res. 30:e36. doi: 10.1093/nar/30.9.e36

Pfaffl, M. W., Tichopad, A., Prgomet, C., and Neuvians, T. P. (2004). Determination of stable housekeeping genes, differentially regulated target genes and sample integrity: bestkeeper-excel-based tool using pair-wise correlations. Biotechnol. Lett. 26, 509-515. doi: 10.1023/B:BILE.0000019559.84305.47

Qutob, D., Hraber, P. T., Sobral, B. W., and Gijzen, M. (2000). Comparative analysis of expressed sequences in Phytophthora sojae. Plant Physiol. 123, 243-254. doi: 10.1104/pp.123.1.243

Reinhart, B. J., Weinstein, E. G., Rhoades, M. W., Bartel, B., and Bartel, D. P. (2002). MicroRNAs in plants. Genes Dev. 16, 1616-1626. doi: $10.1101 /$ gad.1004402

Ronquist, F., and Huelsenbeck, J. P. (2003). MrBayes 3: Bayesian phylogenetic inference under mixed models. Bioinformatics 19, 1572-1574. doi: 10.1093/bioinformatics/btg180

Rozen, S., and Skaletsky, H. (2000). Primer3 on the WWW for general users and for biologist programmers. Methods Mol. Biol. 132, 365-386. doi: 10.1385/159259-192-2:365

Schauer, S. E., Jacobsen, S. E., Meinke, D. W., and Ray, A. (2002). DICER-LIKE1: blind men and elephants in Arabidopsis development. Trends Plant Sci. 7, 487-491. doi: 10.1016/S1360-1385(02)02355-5

Shi, H., Tschudi, C., and Ullu, E. (2006). An unusual Dicer-like1 protein fuels the RNA interference pathway in Trypanosoma brucei. RNA 12, 2063-2072. doi: 10.1261/rna.246906

Soifer, H. S., Sano, M., Sakurai, K., Chomchan, P., Saetrom, P., Sherman, M. A., et al. (2008). A role for the Dicer helicase domain in the processing of thermodynamically unstable hairpin RNAs. Nucleic Acids Res. 36, 6511-6522. doi: 10.1093/nar/gkn687

Tabara, H., Sarkissian, M., Kelly, W. G., Fleenor, J., Grishok, A., Timmons, L., et al. (1999). The rde-1 gene, RNA interference, and transposon silencing in C. elegans. Cell 99, 123-132. doi: 10.1016/S0092-8674(00)81644-X

Tahbaz, N., Kolb, F. A., Zhang, H., Jaronczyk, K., Filipowicz, W., and Hobman, T. C. (2004). Characterization of the interactions between mammalian
PAZ PIWI domain proteins and Dicer. EMBO Rep. 5, 189-194. doi: 10.1038/sj.embor.7400070

Tyler, B. M. (2007). Phytophthora sojae: root rot pathogen of soybean and model oomycete. Mol. Plant Pathol. 8, 1-8. doi: 10.1111/j.1364-3703.2006. 00373.x

Tyler, B. M., Tripathy, S., Zhang, X., Dehal, P., Jiang, R. H. Y., Aerts, A., et al. (2006). Phytophthora genome sequences uncover evolutionary origins and mechanisms of pathogenesis. Science 313, 1261-1266. doi: 10.1126/science.1128796

Tyler, B. M., Wu, M., Wang, J., Cheung, W., and Morris, P. F. (1996). Chemotactic preferences and strain variation in the response of Phytophthora sojae zoospores to host isoflavones. Appl. Environ. Microbiol. 62, 2811-2817.

Vagin, V. V., Sigova, A., Li, C., Seitz, H., Gvozdev, V., and Zamore, P. D. (2006). A distinct small RNA pathway silences selfish genetic elements in the germline. Science 313, 320-324. doi: 10.1126/science.1129333

van West, P., Kamoun, S., van't Klooster, J. W., and Govers, F. (1999). Internuclear gene silencing in Phytophthora infestans. Mol. Cell 3, 339-348.

Vetukuri, R. R., Åsman, A. K. M., Tellgren-Roth, C., Jahan, S. N., and Reimegård, J., Fogelqvist, J., et al. (2012). Evidence for small RNAs homologous to effectorencoding genes and transposable elements in the oomycete Phytophthora infestans. PLoS ONE 7:e51399. doi: 10.1371/journal.pone.0051399

Vetukuri, R. R., Avrova, A. O., Grenville-Briggs, L. J., Van West, P., Söderbom, F., Savenkov, E. I., et al. (2011). Evidence for involvement of Dicer-like, Argonaute and histone deacetylase proteins in gene silencing in Phytophthora infestans. Mol. Plant Pathol. 12, 772-785. doi: 10.1111/j.1364-3703.2011. 00710.x

Wightman, B., Ha, I., and Ruvkun, G. (1993). Posttranscriptional regulation of the heterochronic gene lin-14 by lin- 4 mediates temporal pattern formation in $C$. elegans. Cell 75, 855-862. doi: 10.1016/0092-8674(93)90530-4

Xie, Z., Allen, E., Wilken, A., and Carrington, J. C. (2005). DICER-LIKE 4 functions in trans-acting small interfering RNA biogenesis and vegetative phase change in Arabidopsis thaliana. Proc. Natl. Acad. Sci. U.S.A. 102, 12984-12989. doi: 10.1073/pnas.0506426102

Xie, Z., Johansen, L. K., Gustafson, A. M., Kasschau, K. D., Lellis, A. D., Zilberman, D., et al. (2004). Genetic and functional diversification of small RNA pathways in plants. PLoS Biol. 2:E104. doi: 10.1371/journal.pbio.0020104

Yu, D., Fan, B., MacFarlane, S. A., and Chen, Z. (2003). Analysis of the involvement of an inducible Arabidopsis RNA-dependent RNA polymerase in antiviral defense. Mol. Plant Microbe Interact. 16, 206-216. doi: 10.1094/MPMI.2003.16.3.206

Zamore, P. D., Tuschl, T., Sharp, P. A., and Bartel, D. P. (2000). RNAi: double-stranded RNA directs the ATP-dependent cleavage of mRNA at 21 to 23 nucleotide intervals. Cell 101, 25-33. doi: 10.1016/S0092-8674(00) 80620-0

Zong, J., Yao, X., Yin, J., Zhang, D., and Ma, H. (2009). Evolution of the RNAdependent RNA polymerase (RdRP) genes: duplications and possible losses before and after the divergence of major eukaryotic groups. Gene 447, 29-39. doi: 10.1016/j.gene.2009.07.004

Conflict of Interest Statement: The authors declare that the research was conducted in the absence of any commercial or financial relationships that could be construed as a potential conflict of interest.

Copyright (C) 2016 Bollmann, Fang, Press, Tyler and Grünwald. This is an open-access article distributed under the terms of the Creative Commons Attribution License (CC $B Y)$. The use, distribution or reproduction in other forums is permitted, provided the original author(s) or licensor are credited and that the original publication in this journal is cited, in accordance with accepted academic practice. No use, distribution or reproduction is permitted which does not comply with these terms. 